\title{
Public Value - Gesellschaftliche Wertschöpfung als unternehmerische Pflicht
}

\author{
Peter Gomez und Timo Meynhardt
}

Freies Unternehmertum lässt sich nicht dadurch rechtfertigen, dass es gut ist für das Geschäft. Es legitimiert sich nur durch seinen gesellschaftlichen Nutzen.

Peter Drucker, 1973

Management - eine unverstandene gesellschaftliche Funktion.

Hans Ulrich, 1983

\section{Wertschöpfung im Spannungsfeld von Unternehmensinteressen und öffentlichen Erwartungen}

Peter Drucker und Hans Ulrich haben die moderne Managementlehre in ihrer ganzheitlichen Ausrichtung entscheidend geprägt: der eine als Pionier in der angelsächsischen Welt, der andere als Wegbereiter im deutschsprachigen Europa. Beide vereinte der Gedanke, dass

Dieser Aufsatz basiert auf der Abschiedsvorlesung von Peter Gomez, gehalten am 23.Oktober 2012 an der Universität St. Gallen. Er fasst die wesentlichen Argumente in pointierter Form zusammen.

P. Gomez $(\bowtie)$

Center for Leadership and Values in Society, Universität St. Gallen (HSG),

Dufourstrasse 40a, 9000 St. Gallen, Schweiz

E-Mail: peter.gomez@unisg.ch

T. Meynhardt

Center for Leadership and Values in Society, Universität St. Gallen (HSG),

Dufourstrasse 40a, 9000 St. Gallen, Schweiz

E-Mail: timo.meynhardt@unisg.ch

Leuphana Universität Lüneburg, Scharnhorststraße 1, 21335 Lüneburg, Deutschland

E-Mail: timo.meynhardt@uni.leuphana.de

C. von Müller, C.-P. Zinth (Hrsg.), Managementperspektiven für die Zivilgesellschaft des 21 . 

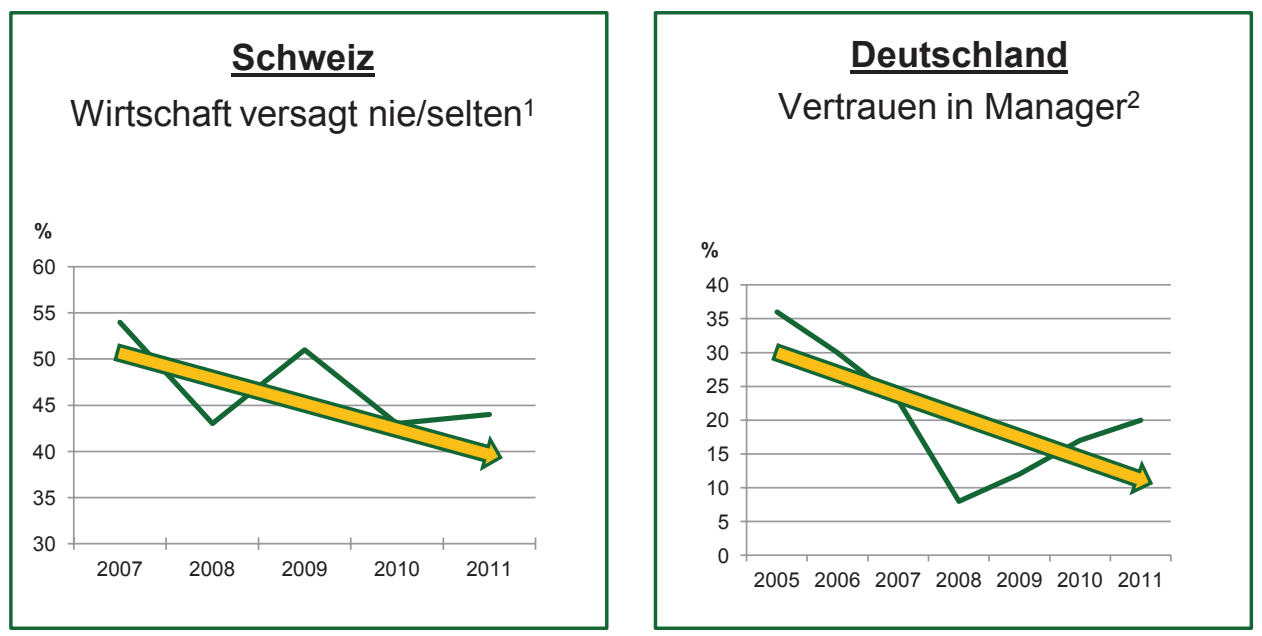

Abb. 1 Vertrauensbarometer Schweiz und Deutschland (2005-2011). 1) gfs.bern (2011). Credit Suisse Sorgenbarometer 2011. 2) GfK Custom Research (2005-2011). GfK Global Trust INDEX

sich Unternehmensführung letztlich über einen gesellschaftlichen Nutzen legitimieren muss. Sie entwickelten ihre Ideen und Konzepte in einer Zeit, in der die Betriebswirtschaftslehre die Standards vor allem durch eine funktionale Betrachtung ihrer Teildisziplinen wie Beschaffung, Produktion, Marketing, Rechnungslegung, Personal und Organisation setzte. Diese Binnensicht hatte einen Preis: Dem Unternehmensumfeld wurde wenig Beachtung geschenkt. Die beiden Managementdenker waren insofern ihrer Zeit weit voraus und sind heute noch wegweisend für ganzheitliches Denken und Handeln im Management.

Die Ausrichtung unternehmerischer Tätigkeit auf die gesellschaftlichen Bedürfnisse und Erwartungen ist in den vergangenen Jahren der Finanz- und Wirtschaftskrise immer mehr in den Fokus gerückt. Die Gesellschaft fordert eine Auseinandersetzung zunehmend von den Unternehmen ein, sie lässt sich nicht mehr mit der „Sachlogik“ des Wirtschaftens oder dem Aktionärsnutzen abspeisen. Dies stellt die Unternehmensführung vor große Herausforderungen, ganz besonders was die Einschätzung der eigenen Leistung anbelangt. Dies dokumentieren die folgenden empirischen Erhebungen eindrücklich.

Das Center for Leadership and Values in Society (CLVS) der Universität St. Gallen hat 40 oberste Führungskräfte von Unternehmen und Organisationen der Privatwirtschaft, der öffentlichen Verwaltung und des Non-Profitsektors nach deren Beitrag zur gesellschaftlichen Wertschöpfung befragt. Dass sich dabei je nach Sektor und in Abhängigkeit von Turbulenzen im Umfeld unterschiedliche Einschätzungen ergaben, ist nicht weiter erstaunlich. Dass aber fast zwei Drittel der Führungskräfte der Meinung waren, ihr Unternehmen befände sich in völligem Einklang mit den Anliegen der „breiten Öffentlichkeit“, ist schon erstaunlich und bedarf einer vertieften Betrachtung (Gomez und Meynhardt 2012; Meynhardt 2012a).

Wie man es auch wendet: Diese Idealisierung des gesellschaftlichen Wertbeitrages der eigenen Unternehmung passt schwerlich zu den seit Jahren abnehmenden Vertrauenswerten, wie es sich aus den jeweiligen Vertrauensbarometern der Schweiz und Deutschlands herauslesen lässt (Abb. 1). 


\section{What is the purpose of business?}

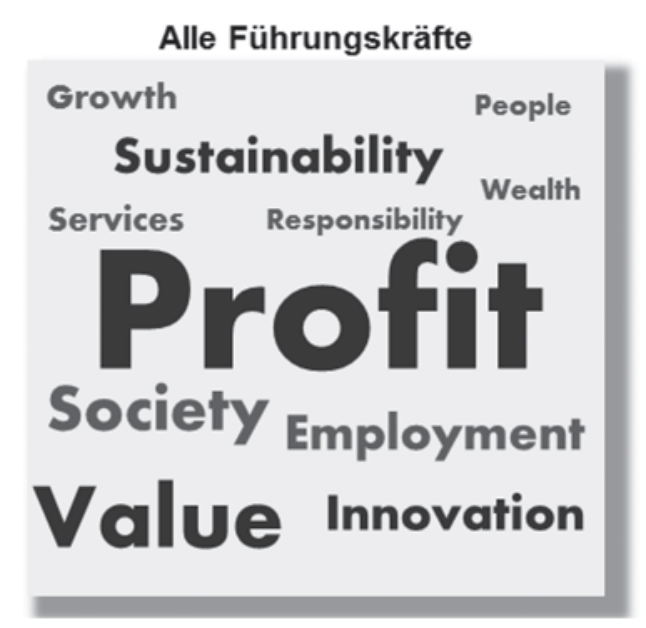

Führungskräfte $<30$ Jahre

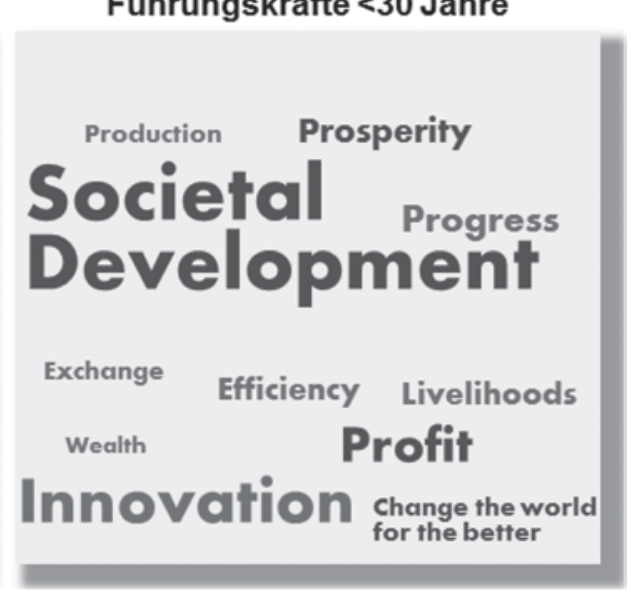

Abb. 2 Wertvorstellungen von Führungskräften. (in Anlehnung an: Deloitte 2012)

Sicher kann man andere Untersuchungen herbeizitieren und neue Belege finden, aber im Trend findet sich eine gewachsene Diskrepanz zwischen Selbst- und Fremdsicht. Zumindest für die Wirtschaft bleibt festzuhalten: Der selbst wahrgenommene Erfolg bei der Erfüllung gesellschaftlicher Bedürfnisse wird überschätzt. Doch auch hier ist Bewegung drin. Zumindest zeigen Untersuchungen zu den Wertvorstellungen von Führungskräften auf, dass bei den Unter-30-Jährigen die Thematik an Bedeutung gewinnt. Während aktive Führungskräfte dem Profitmotiv oberste Priorität einräumen, stellt die jüngere Generation die gesellschaftliche Entwicklung in den Mittelpunkt (Abb. 2).

Es wäre fatal, würde man diesen Befund allein an noch mangelndem Realitätssinn der jungen Generation festmachen. Vielmehr sehen wir darin einen ermutigenden Befund für die Bereitschaft, das Zusammenspiel von Unternehmen und ihrem gesellschaftlichen Umfeld als Teil der Managementaufgabe zu begreifen. Dies ist allerdings leichter gesagt als getan.

\section{Wertschöpfung im Lichte führender Managementkonzepte}

Die Wirtschaft folgt aber heute immer noch meist Spielregeln, die das Eigeninteresse des Unternehmens in den Mittelpunkt stellen. Entsprechend sind ihre Messgrößen eindimensional auf den finanziellen Erfolg oder die Position im Wettbewerb ausgerichtet. Einen wesentlichen Anteil an dieser Entwicklung haben Konzepte, wie sie von Business Schools und Beratungsfirmen entwickelt wurden und die zu weltweiten Standards geworden sind.

Diese Entwicklung fällt vor allem dann ins Auge, wenn man die Betonung des Profitmotivs ins Verhältnis setzt mit der jeweiligen Rolle der gesellschaftlichen Wertschöpfung, also dem Beitrag zum Gemeinwohl. Die folgende Darstellung (Abb. 3) zeigt in vereinfach- 


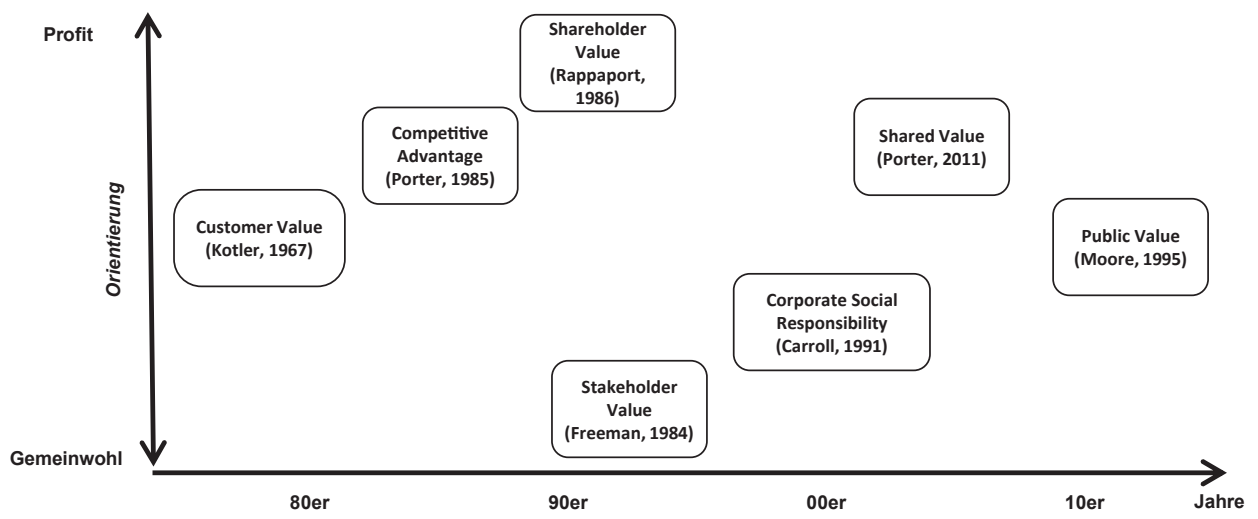

Abb. 3 Messgrößen unternehmerischen Erfolgs

ter Weise die Entwicklung der Messgrößen unternehmerischen Erfolgs über die letzten 40 Jahre.

Die 70er Jahre sahen den Aufstieg des Marketings und damit die Ausrichtung auf den „Customer Value“ oder Kundennutzen. Anfangs der 80er Jahre brachen die großen Zeiten der Wettbewerbsstrategie an mit ihrer Ausrichtung auf den „Competitive Advantage“ im Sinne von Erfolgspositionen im Konkurrenzkampf. Dieser folgte im Übergang zu den 90er Jahren der "Shareholder Value“ oder Aktionärsnutzen mit seinem Fokus auf die finanzielle Leistungsfähigkeit. Die Gegenbewegung in Form des „Stakeholder Value“ oder der Berücksichtigung legitimer Ansprüche ließ nicht lange auf sich warten. Zu Beginn dieses Jahrhunderts rückte verstärkt die "Corporate Social Responsibility“ in den Mittelpunkt des Interesses, die schädliche Nebenwirkungen unternehmerischen Handelns abdämpfen sollte. Das zurzeit aktuellste Konzept trägt den Namen „Shared Value“ und fordert Unternehmen dazu auf, die eigene Leistungssteigerung mit ihrem Umfeld zu teilen. Mit dem Konzept des "Public Value" oder gesellschaftlichen Nutzens präsentierte sich zu Beginn dieses Jahrhunderts, zunächst im Kontext der öffentlichen Verwaltung, ein Ansatz, der Wertschöpfung von der Gesellschaft her definiert und ein mehrdimensionales Wertschöpfungsverständnis zugrunde legt.

Alle diese Konzepte haben unzweifelhaft ihre Verdienste, indem sie wichtige Aspekte des Managements vertieften und entsprechende Erkenntnisse einem breiten Kreis zugänglich machen. Sie zeichnen sich aber durch zwei Eigenschaften aus, die sich bei unreflektierter Umsetzung als gefährlich erweisen können: Ihre Eindimensionalität und ihre „Inside-Out“ Perspektive. Einerseits wird das Unternehmensgeschehen auf ein Thema reduziert, das nach allen Regeln der Kunst durchdekliniert wird und dabei wesentliche andere Aspekte ausblendet. Anderseits wird meistens eine Inside-Out Perspektive eingenommen: Die Unternehmen geben vor zu wissen, was die breite Öffentlichkeit oder einzelne Anspruchsgruppen als wertvollen gesellschaftlichen Beitrag verstehen. Sie erachten es nicht 
als notwendig, dies selbst bei den Betroffenen in Erfahrung zu bringen. Die Finanz- und Wirtschaftskrise hat gezeigt, dass dies nicht mehr länger akzeptiert wird, die Frage der Legitimität unternehmerischen Handelns wird öffentlich immer lauter gestellt.

Das CLVS an der Universität St. Gallen hat sich zum Ziel gesetzt, die von Peter Drucker und Hans Ulrich entwickelte Sicht der gesellschaftlichen Verantwortung von Unternehmen weiter zu entwickeln. Neben intensiver Grundlagenforschung zählt dazu auch die Übertragung neuester Erkenntnisse verwandter Disziplinen (z. B. Psychologie und Soziologie) in die Managementperspektive. Dies einerseits aus einer mehrdimensionalen Perspektive und anderseits mit einem expliziten „Outside-in“ Fokus. „Die Gesellschaft sitzt immer mit am Tisch“ - diese Aussage soll mit Inhalt gefüllt werden. Die damit verbundene erweiterte Wertschöpfungsperspektive hat ihre Vorläufer im „Wertmanagement“ (Gomez 1993). Gewissermaßen in zweiter Generation (Meynhardt 2012b, 319 ff) geht es nun darauf aufbauend stärker um den Wert für die Gesellschaft.

Dreh- und Angelpunkt ist die Idee des Public Value (Meynhardt und Gomez 2013; Meynhardt 2009). Diese erfasst das Phänomen, dass jede Organisation auf das Einfluss nimmt, was der Einzelne als „Gesellschaft“ erlebt. Dies nennen wir gesellschaftliche Wertschöpfung! Nicht abstrakte Konzepte und Zahlenwerke schaffen Wert; dieser muss in der Lebenswelt des Einzelnen erfahrbar werden. „Public Value wird erst dann geschaffen oder zerstört, wenn das individuelle Erleben und Verhalten von Personen und Gruppen so beeinflusst wird, dass dies stabilisierend oder destabilisierend auf Bewertungen des gesellschaftlichen Zusammenhalts, das Gemeinschaftserleben und die Selbstbestimmung des Einzelnen im gesellschaftlichen Umfeld wirkt.“ (Meynhardt 2008, 462)

Damit werden menschliche Grundbedürfnisse zum Maßstab des Handelns, an denen sich auch Unternehmen messen lassen müssen. Was sonst könnte der legitime Bezugsrahmen unternehmerischer Freiheit sein?

Eine solche Verankerung der Wirtschaft im sozialen und kulturellen Kontext muss offen sein für Wertepluralität und sich verändernde Erwartungen. Deshalb wird zwar zwischen Grundbedürfnissen differenziert, ohne diese jedoch in eine Hierarchie zu bringen (Meynhardt 2009):

1. Bedürfnis nach Orientierung und Kontrolle (instrumentell-utilitaristisch, Fokus auf den Nutzen)

2. Bedürfnis nach Selbstwerterhalt und -steigerung (moralisch-ethisch, Fokus auf das Individuum)

3. Bedürfnis nach positiven Beziehungen (politisch-sozial, Fokus auf die Gruppe)

4. Bedürfnis nach Unlustvermeidung und Lustgewinn (hedonistisch-ästhetisch, Fokus auf positive Erfahrung)

Übersetzt man diese psychologische Basis in die Unternehmenswelt, so entsteht ein mehrdimensionales, nicht-normatives Bezugsystem für legitimes unternehmerisches Handeln. Die folgende Darstellung (Abb. 4) zeigt die daraus resultierende Public Value Scorecard (Meynhardt 2013). 


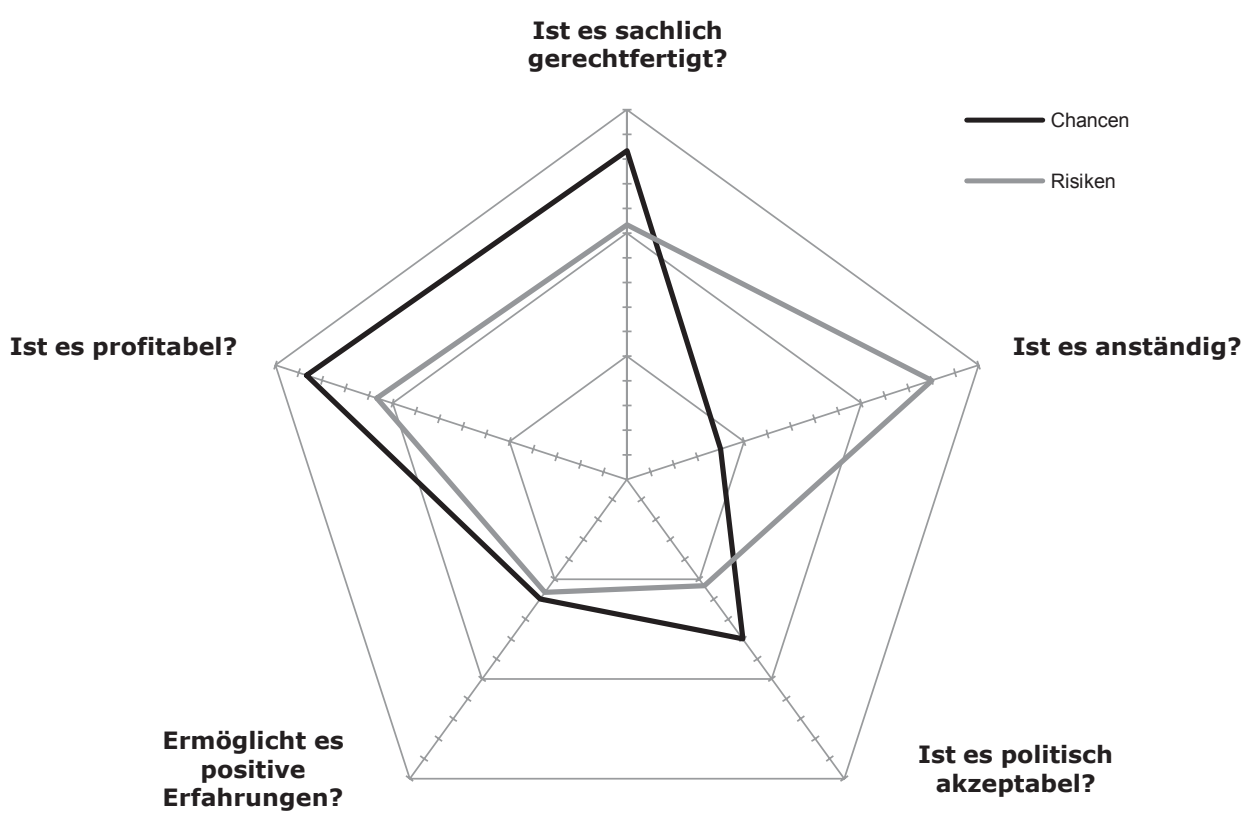

Abb. 4 Die Public Value Scorecard

$\mathrm{Ob}$ man will oder nicht: Jede unternehmerische Aktivität kann anhand dieser fünf Kriterien beurteilt werden. Dabei ist kein Kriterium a priori einem anderen über- oder untergeordnet. Erst bei der Ableitung von Handlungsalternativen können die Entscheidungsträger eine Gewichtung vornehmen. Damit sind die Voraussetzungen geschaffen, die gesellschaftliche Wertschöpfung von Unternehmen zu erfassen.

\section{Wertschöpfung ganzheitlich erfassen und beurteilen}

Das wohl in der Praxis verbreitetste Instrument einer mehrdimensionalen Erfassung der Unternehmensleistung ist die Balanced Scorecard (Kaplan und Norton 1992). Diese misst den Erfolg des Unternehmens aus vier Perspektiven: Finanzen, Kunden, interne Prozesse und Lern-/Wachstumspotentiale. Sie strebt eine ganzheitliche Beurteilung an, kann aber diesen Anspruch in zweifacher Hinsicht nicht erfüllen. Zum einen wird dem Zusammenspiel der einzelnen Perspektiven nicht Rechnung getragen. Auch wenn die einzelnen Unternehmensgrößen durch (kaum qualifizierte) Pfeile verbunden werden, so ist dies noch keine geeignete Erfassung der Dynamik des Unternehmens, sondern lediglich eine Momentaufnahme. Zum andern zeichnet sich die Balanced Scorecard durch eine InsideOut Sicht aus, die Umfeldeinflüsse werden kaum in die Betrachtung einbezogen.

Der Public Value-Ansatz des CLVS an der Universität St. Gallen nimmt die Grundidee der Balanced Scorecard auf, erweitert diese aber um die Outside-In Sicht und die Dyna- 


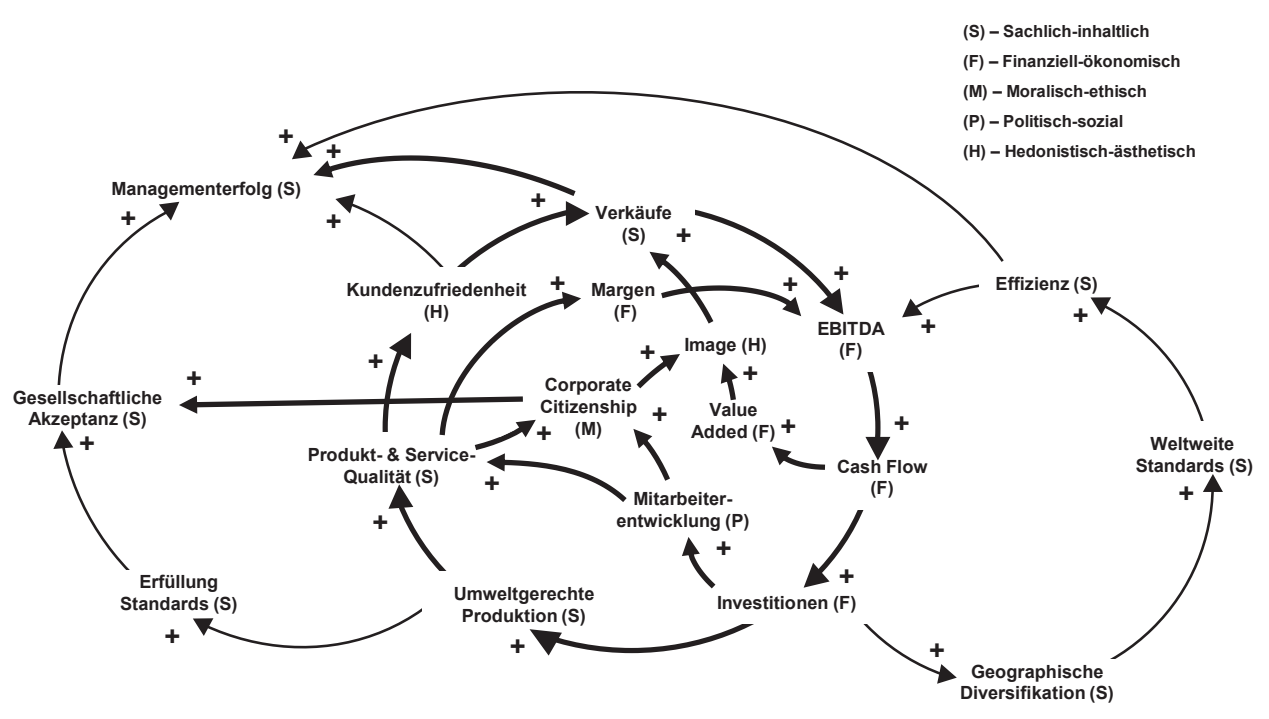

Abb. 5 Netzwerk des Public Value

misierung. Das Erste wird durch die Public Value Scorecard erreicht, das Zweite durch die Methodik des Vernetzten Denkens (Gomez und Probst 1999).

Die Public Value Scorecard setzt das in Abb. 4 vorgestellte Konzept in ein für die Unternehmenspraxis geeignetes Instrument um. Dazu existieren heute vier Erhebungsvarianten, welche jeweils zu Chancen- und Risikoprofilen führen. Je nach Anliegen werden unterschiedlichste interne und externe Gruppen und Stakeholder einbezogen. Diese werden nicht nur im Unternehmen selber, sondern in dessen relevanten Umfeldern gesucht. Damit wird das Versprechen einer wahren Outside-In Sicht eingelöst. Was als „wertvoll“ angesehen wird, bestimmt nicht nur das Unternehmen, sondern die breitere Öffentlichkeit kann ihre Bedürfnisse und Erwartungen authentisch einbringen.

Die Chancen- und Risikoprofile der Public Value Scorecard (Abb. 4) helfen den Führungskräften, Stärken, „Blinde Flecken“ und mögliche Schwachstellen unternehmerischer Aktivitäten zu identifizieren. Welche Maßnahmen sollen nun aber ergriffen werden, und wie sind die entsprechenden Erfolgsaussichten? Hier kommt nun die Methodik des Vernetzten Denkens zum Zuge. Diese verbindet die kritischen Größen der einzelnen Perspektiven des Public Value in Form von Kreisläufen der gegenseitigen Beeinflussung. In Klammern ist jeweils die besonders in Frage stehende Public Value-Dimension gekennzeichnet. Während es beispielsweise beim EBITDA in erster Linie um eine finanziell-ökonomische Betrachtung geht, steht bei der Corporate Citizenship die moralische Komponente im Vordergrund. Die Pfeile haben eine eindeutige Denotation, sie benennen eine verstärkende (+) oder eine stabilisierende (-) Wirkung. Weiter geben sie die zeitliche Verzögerung an, mit der Eingriffe in das System wirken (Abb. 5). 


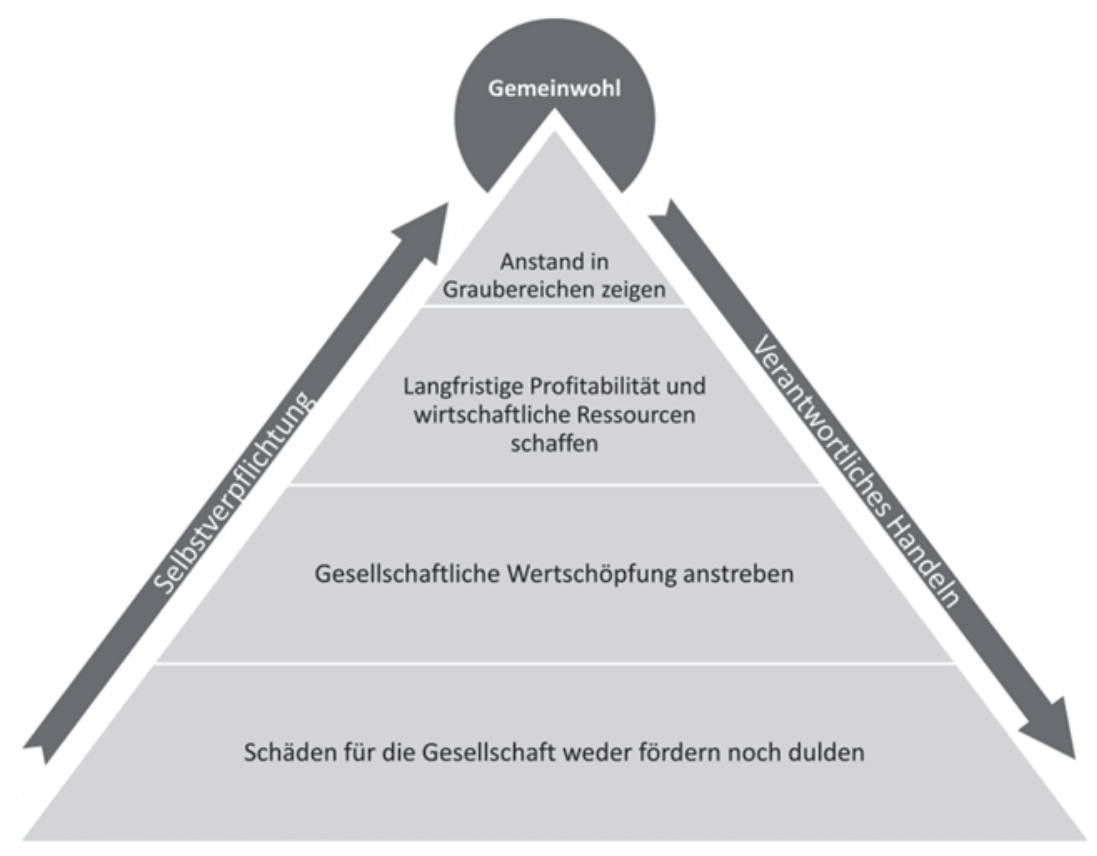

Abb. 6 Gemeinwohlpyramide des Schweizer Dialogs

Mit Hilfe eines solchen Netzwerks lassen sich nicht nur die optimalen Ansatzpunkte zur Beeinflussung des Public Value identifizieren. Das Netzwerk eignet sich ebenso für die Entwicklung möglicher Umfeldszenarien und zur Bestimmung von Frühwarnindikatoren. Die Ergebnisse der Public Value Scorecard werden auf diese Weise im dynamischen Zusammenhang verstehbar und vor allem wird ihre wechselseitige Abhängigkeit sichtbar.

\section{Wertschöpfung kommunizieren}

Die bisherigen Überlegungen haben sich mit der Bereitstellung eines umfassenden Bezugsrahmens und der Entwicklung eines praxistauglichen Instrumentariums zur Gestaltung des Public Value befasst. Die Botschaft muss aber auch bei den unmittelbar Betroffenen ankommen, konkret bei den Bürgerinnen und Bürgern und den verschiedenen Anspruchsgruppen. Das CLVS an der Universität St. Gallen hat hierzu zwei Initiativen gestartet, den Schweizer Dialog und den GemeinwohlAtlas.

Im Höhepunkt der weltweiten Finanzkrise haben sich 30 Schweizer Persönlichkeiten aus unterschiedlichen wirtschaftlichen, politischen und gesellschaftlichen Kreisen zum Dialog mit der Öffentlichkeit zum Thema des „anständigen Wirtschaftens“ entschlossen. Grundlage war die auf Basis der skizzierten Grundlagen entwickelte Verantwortungspyramide (Meynhardt und Gomez, under review) im Sinne einer Verpflichtung auf grundlegende Werte, die bei unternehmerischen Entscheiden handlungsleitend sein sollen (Abb. 6). 


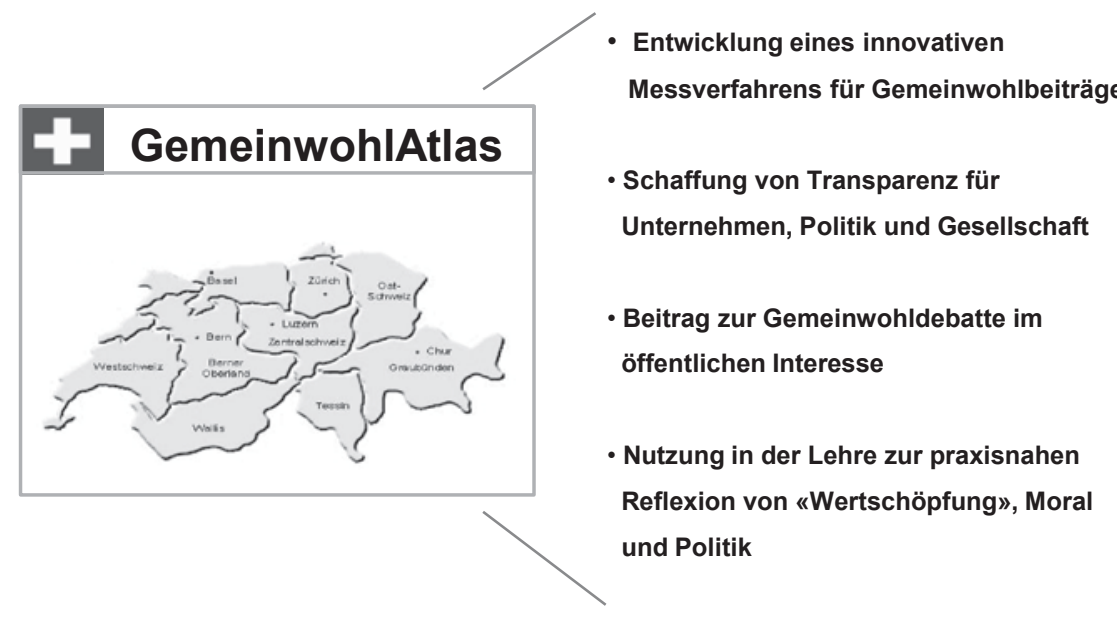

Abb. 7 GemeinwohlAtlas. eigene Darstellung

In einem Blog (www.schweizerdialog.ch) haben sich die Teilnehmenden nicht nur selber verpflichtet, sich in ihren Organisationen von diesen Werten leiten zu lassen. Sie haben sich auch mit Aussagen zur Anwendung dieser Werte im Kontext der Managemententschädigung, der Generationengerechtigkeit und der Nachhaltigkeit der öffentlichen Diskussion gestellt.

Als neue Initiative wurde vor kurzem der GemeinwohlAtlas lanciert. Dieser wird für alle Regionen der Schweiz Daten erheben zu den Gemeinwohlbeträgen von Unternehmen, staatlichen Institutionen und Non-Profit-Organisationen. Die Ziele dieses Atlas sind in Abbildung 7 festgehalten.

Damit soll nicht nur die Debatte zu diesem Thema prominent lanciert werden, sondern die Unternehmen sollen diese Daten auch zur Weiterentwicklung ihrer Strategien nutzen können. Denn wie lautet eine alte Weisheit: „Was gemessen wird, wird auch getan!“

Mit dem vorgestellten Public Value-Konzept und dem darauf basierenden Instrumentarium möchten wir die vielfältigen Spannungen zwischen Unternehmen und Gesellschaft thematisieren und Lösungswege aufzeigen. Dies ist auch notwendig, denn die Öffentlichkeit stellt immer dringlicher die Frage nach der Legitimität unternehmerischen Handelns. Dass etwas „rechtens“, also nicht verboten sei, reicht der Gesellschaft nicht mehr aus. Vielmehr wird der Ruf laut: „So etwas tut man nicht!“ Dass Führungskräfte solche Entwicklungen frühzeitig erkennen und produktiv damit umgehen lernen, ist das oberste Ziel des hier vorgestellten Public Value-Konzepts. 


\section{Literatur}

Carroll AB (1991) The pyramid of corporate social responsibility: towards the moral management of organizational stakeholders. Business Horizons 34(4):39-48

Deloitte (2012) The Millenial Survey 2011. Online unter http://www.deloitte.com/assets/Dcom-Global/Local\%20Assets/Documents/Business\%20Society\%20documents/Millennial_Survey_2011_ web.pdf

Drucker PF (1973) Management: tasks, responsibilities, practices. HarperBusiness Edition, New York

Freeman RE (1984) Strategic management: a stakeholder approach. Pitman, Boston

GfK Custom Research (2005-2011) GfK Global Trust INDEX

gfs.bern (2011) Starker Franken, schwaches Vertrauen: Credit Suisse Sorgenbarometer 2011. Online unter https://www.credit-suisse.com/asset_management/downloads/marketing/report_gfs_ bern_1.pdf

Gomez P, Meynhardt T (2012) More foxes in the boardroom: systems thinking in action. Systemic management for intelligent organizations: concepts, models-based approaches and applications. Springer. In: Groesser, St N, Zeier R (Hrsg) Heidelberg, S 83-98

Gomez P, Probst G (1999) Die Praxis des ganzheitlichen Problemlösens: Vernetzt denken, unternehmerisch handeln, persönlich überzeugen, 3 Aufl. Paul Haupt Verlag

Gomez P (1993) Wertmanagement. Vernetzte Strategien für Unternehmen im Wandel. ECON Verlag, Düsseldorf

Kaplan RS, Norton DP (1992) The balanced scorecard - measures that drive performance. Harvard Bus Rev 70(1) (Januar-Februar):71-79

Kotler P (1967) Marketing Management: Analysis, Planning and Control. Prentice-Hall, EnglewoodCliffs

Meynhardt T (2008) Public Value: Oder was heißt Wertschöpfung zum Gemeinwohl?, der moderne staat - Zeitschrift für Public Policy. Recht und Management 1:457-468

Meynhardt T (2009) Public value inside: what is public value creation? Int J Public Admin 32(3):192219

Meynhardt T (2012a) Mehr Füchse, weniger Igel!. Harvard Bus Manag 7:94-95

Meynhardt T (2012b) Public Value - oder die zweite Generation des Wertmanagements. In: RüeggStürm J, Bieger Th (Hrsg) Unternehmerisches Management. Herausforderungen und Perspektiven. Festschrift für Prof. Dr. Peter Gomez, Haupt, Bern, S 319-324

Meynhardt T (2013) Werkzeugkiste: Public Value Scorecard, OrganisationsEntwicklung. Zeitschrift für Unternehmensentwicklung und Change Management (04):79-83

Meynhardt T, Gomez P (2013) Organisationen schöpfen Wert für die Gesellschaft. In: Heuser J et al (Hrsg) DIE ZEIT erklärt die Wirtschaft. Murmann, S 199-207

Meynhardt T, Gomez P (under review) Do it yourself! Building Blocks for New Pyramids of Corporate Social Responsibilities

Moore MH (1995) Creating public value: strategic management in Government. Harvard University Press, Cambridge

Porter ME (1985) Competitive advantage: creating and sustaining superior performance. Macmillan, New York

Porter ME, Kramer MR (2011) Creating shared value. Harvard Bus Rev 89(1-2):62-77

Rappaport A (1986) Creating shareholder value: the new standard for business performance. Free Press, New York

Ulrich H (1983) Management - eine unverstandene gesellschaftliche Funktion In: Siegwart H, Probst G (Hrsg) Mitarbeiterführung und gesellschaftlicher Wandel. Haupt, Bern, S 133-152 


\section{焦 Springer}

http://www.springer.com/978-3-658-02522-9

Managementperspektiven für die Zivilgesellschaft des 21 .

Jahrhunderts

Management als Liberal Art

Müller, C.; Zinth, C. -P. (Hrsg.)

2014, XIX, 234 S. 35 Abb., Softcover

ISBN: $978-3-658-02522-9$ 